\title{
Characterization of bHLH/HLH genes that are involved in brassinosteroid (BR) signaling in fiber development of cotton (Gossypium hirsutum)
}

\author{
Rui Lư ${ }^{\dagger}$, Jiao Zhang ${ }^{\dagger}$, Dong Liu, Ying-Li Wei, Yao Wang and Xue-Bao Li ${ }^{*}$ (1)
}

\begin{abstract}
Background: Basic helix-loop-helix/helix-loop-helix (bHLH/HLH) transcription factors play important roles in plant development. Many reports have suggested that bHLH/HLH proteins participate in brassinosteroid (BR) hormone signaling pathways to promote cell elongation. Cotton fibers are single-cells and derived from seed surface. To explore the roles of bHLH/HLH proteins in cotton fiber development progress by modulating BR signaling pathway, we performed a systematic analysis of the bHLH/HLH gene family in upland cotton (Gossypium hirsutum) genome.

Results: In this study, we identified 437 bHLH/HLH genes in upland cotton (G. hirsutum) genome. Phylogenetic analysis revealed that GhbHLH/HLH proteins were split into twenty six clades in the tree. These GhbHLH/HLH genes are distributed unevenly in different chromosomes of cotton genome. Segmental duplication is the predominant gene duplication event and the major contributor for amplification of GhbHLH/HLH gene family. The GhbHLH/HLHs within the same group have conserved exon/intron pattern and their encoding proteins show conserved motif composition. Based on transcriptome data, we identified $77 \mathrm{GhbHLH/HLH}$ candidates that are expressed at relatively high levels in cotton fibers. As adding exogenous BR (brassinolide, BL) or brassinazole (Brz, a BR biosynthesis inhibitor), expressions of these GhbHLH/HLH genes were up-regulated or down-regulated in cotton fibers. Furthermore, overexpression of GhbHLH282 (one of the BR-response genes) in Arabidopsis not only promoted the plant growth, but also changed plant response to BR signaling.
\end{abstract}

Conclusion: Collectively, these data suggested that these GhbHLH/HLH genes may participate in BR signaling transduction during cotton fiber development. Thus, our results may provide a valuable reference data as the basis for further studying the roles of these $6 H L H / H L H$ genes in cotton fiber development.

Keywords: Cotton (Gossypium hirsutum), bHLH/HLH transcription factor, Fiber development, Phylogenetic analysis, Brassinosteroid (BR) signaling, Gene expression

\section{Background}

Cotton, one of the most important economic crops, supplies the largest number of natural fibers in the textile market around the world. The cotton genus (Gossypium) is composed of nearly 50 species, of which four species, including two diploids (G. arboreum and $G$. herbaceum, $2 \mathrm{n}=2 \times=26$ AA) and two allotetraploids

\footnotetext{
* Correspondence: xbli@mail.ccnu.edu.cn

${ }^{\dagger}$ Rui Lu and Jiao Zhang contributed equally to this work.

Hubei Key Laboratory of Genetic Regulation and Integrative Biology, School of Life Sciences, Central China Normal University, Wuhan 430079, China
}

(G. hirsutum and G. barbadense, $2 \mathrm{n}=4 \mathrm{x}=52$ AADD), have been widely cultivated for the commercial values of fibers [1]. Upland cotton (G. hirsutum) accounts for the largest planting area among the varieties of cotton crops and provides most of the valuable fibers needed by modern textile industry. Fiber development is divided into four overlapping stages, fiber initiation, elongation, secondary cell wall thickening and maturity [2]. The cotton fiber is regarded as a powerful cell research model since it is an easily isolated single cell with distinct stages of cotton fiber cell development. In

(c) The Author(s). 2018 Open Access This article is distributed under the terms of the Creative Commons Attribution 4.0 International License (http://creativecommons.org/licenses/by/4.0/), which permits unrestricted use, distribution, and reproduction in any medium, provided you give appropriate credit to the original author(s) and the source, provide a link to the Creative Commons license, and indicate if changes were made. The Creative Commons Public Domain Dedication waiver (http://creativecommons.org/publicdomain/zero/1.0/) applies to the data made available in this article, unless otherwise stated. 
vitro ovules culture can be carried out to make further research about detailed development stages of cotton fibers [3, 4].

Basic helix loop helix/helix loop helix (bHLH/HLH) proteins were named for the basic helix loop helix domains. The bHLH domains are comprised of 50-60 amino acids with two functionally different regions, the $\mathrm{N}$ terminal basic region and the $\mathrm{C}$ terminal helix-loophelix region. The basic region, consisting of around 15 amino acids with numerous basic residues, can recognize and bind to DNA $[5,6]$. The HLH region, including two amphipathic $\alpha$-helices that are parted by a loop region with alterable sequence, can form the dimers with other HLH domains [7]. In higher plants, the bHLH/HLH proteins participate in regulation of plant growth and development, such as light signal transmission, plant hormone signals and organs development. For example, in light signal transmission, AtPIF4 takes part in regulating photomorphogenesis mainly by interacting with photochromes [8]. AtSPT is not only related to Arabidopsis sterility, but also has a coordinating relationship with DELLA protein to regulate the expression of gibberellins (GA) response genes jointly. AtSPT mutation can remove its inhibition of cell growth $[9,10]$. AtGL3 is also involved in the development of Arabidopsis trichomes by contributing to form the trichome promoting comlpex TTG1/GL3 (EGL3) /GL1 [11, 12]. In the stem or leaf epidermal cells of Arabidopsis, the complex can induce the expression of the downstream gene GL2 to determine the development of epidermal cells. And the MYB proteins, AtCPC, AtTRY, AtTCL1 and AtETC1, can bind to GL3 competing with GL1, thereby negatively regulating the initiation of trichomes $[13,14]$.

Brassinosteroid (BR) signalling is a well-described signalling pathway in Arabidopsis that plays a crucial role in plant growth and development. Many bHLH/HLH protiens have been reported to be involved in the $B R$ signaling transduction. AtbHLH064 (HBI1), AtbHLH158 (IBH1), AtbHLH044/058/050 (BEE1/2/3), AtbHLH046/ 102/141 (BIM1/2/3), AtbHLH136/135 (PRE1/3) and AtbHLH150/148/147/149 (AIF1/2/3/4) are regulated by BZR1/BES1 to influence BR signaling in Arabidopsis [15-19]. Most of them can form homodimers and heterodimers, endowing them with the capacity to function in the regulation of multiple transcriptional programs [20]. For example, AtCESTA can form heterodimer with AtbHLH044 (BEE1) to regulate the expression of BR biosynthesis related gene CPD positively [21]. AtbHLH064 (HBI1) promotes cell elongation by regulating downstream genes expression and its functions can be inhibited by AtbHLH158 (IBH1), which inhibition can be relieved by the interaction of AtbHLH136 (PRE1) and AtbHLH158 (IBH1) [22, 23]. A report also demonstrates that AtbHLH136 (PRE1), AtbHLH158 (IBH1) and AtbHLH049/074/077 (ACE1/2/3) constitute a triantagonistic bHLH system that competitively regulates cell elongation [24, 25].

Exogenous application of BL (BR) promotes cotton fiber cell elongation while treatment of cotton floral buds with Brz (a BR inhibitor) results in the complete absence of fiber cell differentiation, indicating that BR is required for fiber initiation and elongation [26, 27]. Besides, our previous study indicated that Gh14-3-3 proteins are involved in regulating fiber initiation and elongation through their interacting with GhBZR1 to modulate BR signalling [28]. Also, cotton bHLH/HLH transcription factors may play important roles in fiber development [29-31]. However, little is known about how bHLH/HLH transcription factors modulate BR signaling during fiber development of cotton in detail so far. In our study, we genome-widely identified the cotton $b H L H / H L H$ genes that may be involved in BR signaling in fiber development. Furthermore, the characters of these cotton bHLH/HLH transcription factors were approached in detail.

\section{Results}

Characterization of cotton bHLH/HLH transcription factors To identify the $b H L H / H L H$ transcription factor genes in upland cotton (G. hirsutum) genome, all published $b H L H /$ $H L H$ gene sequences of Arabidopsis and rice were employed as queries to perform homologous blast searches against the cotton genome database (https:// www.cottongen.org/tools/blast/blast) [32]. Originally, 498 candidate bHLH/HLH genes were identified in cotton. Among them, 61 repeated sequences were discarded. Furthermore, to evaluate the reliability of the initial results, the conserved bHLH/HLH domain of predicted bHLH/ HLH proteins were confirmed using the Hmmscan program and the pfam tools (http://www.ebi.ac.uk/Tools/ hmmer/search/hmmscan) according to reported methods [33]. The results showed that all the 437 putative genes have conserved bHLH or HLH domains in their sequences. Since there was no uniform annotation for the $G h b H L H / H L H$ genes, the $b H L H / H L H$ genes are named as GhbHLHOO1 to GhbHLH437 according to their chromosome locations. The average length of the newly identified bHLH/HLH proteins is 353 amino acids with variation range of 73 to 1302 . The characters of the bHLH/HLH proteins including the amino acid numbers, molecular weights (MW), theoretical isoelectric points (pI) and chromosome locations were listed in Additional file 1: Table S1.

\section{Phylogenetic relationship of cotton bHLH/HLH proteins}

To exmaine the evolutionary history and phylogenetic relationship of upland cotton bHLH/HLH proteins, an 
unrooted phylogenetic tree was constructed using the Neighbor Joining (NJ) method based on the results of multiple sequence alignment of the identified 437 GhbHLH/HLH domain sequences with 172 Arabidopsis bHLH/HLH domain sequences and one rice bHLH domain sequence (Additional file 1: Figure S1). To verify the reliability of the constructed phylogenetic tree, the Maximum likelihood, Minimal Evolution and PhyML methods were employed to reconstruct the phylogenetic trees of bHLH/HLH transcription factors respectively. The phylogenetic trees constructed by the four methods were identical with only slight differences in some branches. Another unrooted phylogenetic tree was also constructed using the Neighbor Joining (NJ) method based on the results of multiple sequence alignment of the 437 GhbHLH/HLH domain sequences (Fig. 1). Subsequently, the analyses of gene structures, motif sites distribution and gene expression patterns also confirmed the validity of the phylogenetic relationship of GhbHLH/HLH protein sequences.

On the basis of the NJ phylogenetic tree, the 610 bHLH/HLH protein sequences were grouped into 26 subfamilies (including S1-S5, S7a, S7b, S9-S18 and S23-S31) (Additional file 1: Figure S1), which remain in the classification of bHLH/HLH transcription factors by Lorenzo Carretero-Paulet [34]. Additionally, S7 and
S12 were split into two subgroups (S7a and S7b, S12a and S12b), while S18, S19, S20, S21 and S22 were clustered into a single subfamily in the tree. S25, the largest group among the whole gene family groups, is composed of $61 \mathrm{bHLH} / \mathrm{HLH}$ members, occupying 14\% of the total bHLH/HLH numbers, but S18 contain 2 members, being the smallest clade in the tree (Additional file 1: Table S2). The interspersed distribution of the bHLH/HLH proteins in most clades of the tree implied that the bHLH/HLH proteins expanded before the divergence of the lineages. Most of Arabidopsis $b H L H / H L H$ genes have two or more correspondences in upland cotton genome, indicating that the divergence of cotton and Arabidopsis occurred before GhbHLH/HLH genes duplication. In subfamily 8 , neither GhbHLH/HLH nor AtbHLH/HLH sequences was classified into rice $b H L H / H L H$ sequences, which implied that this group may be lost after the divergence of monocots and dicots.

\section{Chromosomal distribution and duplication of cotton bHLH/HLH genes}

To clarify the chromosomal distribution of the GhbHLH/HLH genes, the physical locations of all the GhbHLH/HLH genes were acquired through the blastn searches against upland cotton genome databases. Among the 437 genes, a total of 397 genes are unevenly

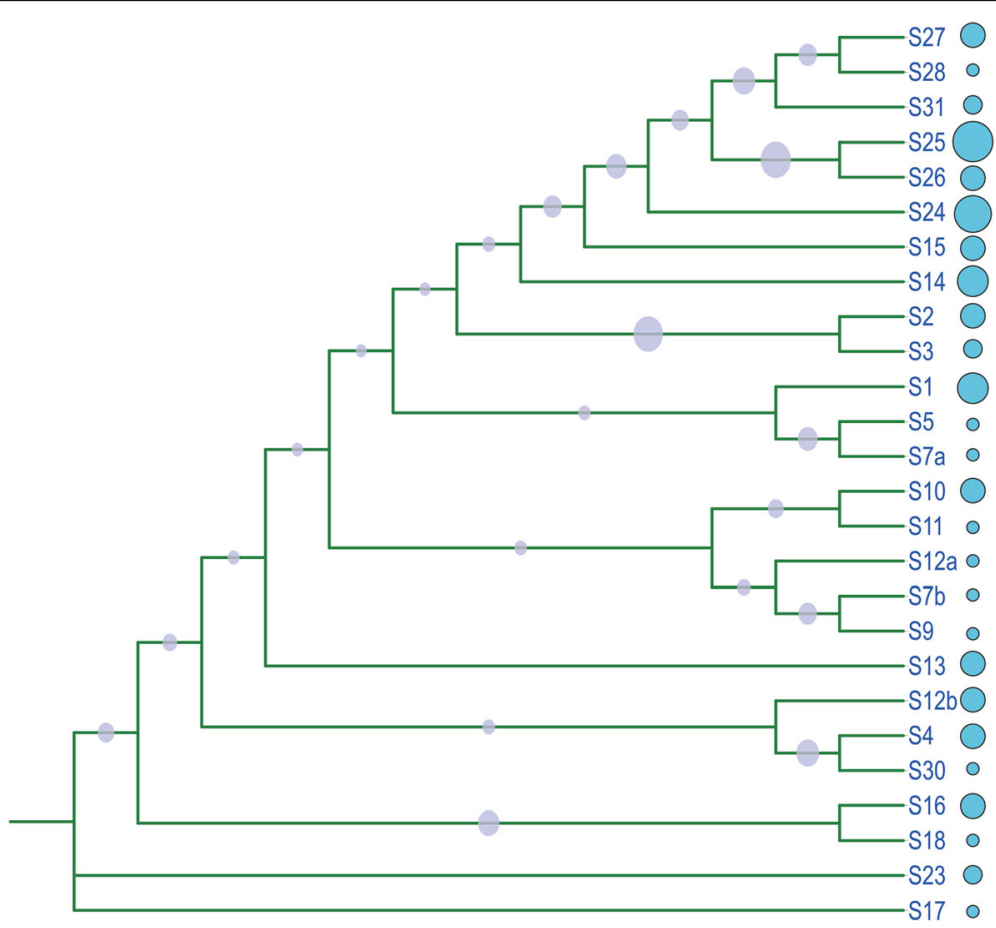

Fig. 1 Phylogenetic relationship of cotton bHLH/HLH transcription factors. MEGA 6.0 software was employed to construct an unrooted phylogenetic tree based on alignments of $437 \mathrm{bHLH} / \mathrm{HLH}$ domains identified from upland cotton (G. hirsutum) using the NeighborJoining (NJ) method with the following parameters: The number of differences model, pairwise deletion and 1000 bootstraps. Subfamilies are collapsed and represented as grey dots with area proportional to member numbers of each subfamily 
distributed on different chromosomes of upland cotton genome, while the remaining 40 genes were unmapped on scaffolds. Upland cotton (G. hirsutum) genome includes A-subgenome and D-subgenome, containing 205 $b H L H / H L H$ and $224 b H L H / H L H$ genes, respectively. In our study, the genes distribution event on A-genomes is similar to that in D-genome. For example, Chromosome A11 of A-subgenome has the largest number of bHLH/HLH genes, in accordance with the fact that Chromosome D11 of D-subgenome has the largest number of $b H L H / H L H$ genes. At the same time, the fewest number of $b H L H / H L H$ genes were found on A1 and D1 chromosomes (Fig. 2).

To clear the clouds on the mechanism behind the expansion of GhbHLH/HLH gene family, gene duplication events about the evolution of upland cotton genome were analyzed. 19 gene pairs in A-subgenome and 21 pairs in D-subgenome were identified respectively. These gene pairs were gathered in the same clade of the phylogenetic tree with high similarity. For example, the sequence of GhbHLHO13 shares 94.5\% sequence similarity with the sequence of GhbHLH127. Among these paralogous gene pairs, 18 pairs in A-subgenome and 19 pairs in D-subgenome are located on different chromosomes, indicating that segmental duplication event plays the leading role in $b H L H / H L H$ gene family amplification during evolution, and no tandem duplication event was observed in these identified gene pairs (Fig. 2).

\section{Structures and conserved motifs of cotton $b H L H / H L H$ genes}

For further insights into the evolutionary relationships of $G h b H L H / H L H$ genes, we observed the exon/intron structures of GhbHLH/HLH genes by alignment of the genomic DNA sequences of these genes with their corresponding cDNA sequences. Another unrooted phylogenetic tree was constructed to determine if the exon/ intron pattern is consistent with the phylogenetic classification. As expected, most GhbHLH/HLH genes in the same group show similar exon/intron pattern in terms of exon length and intron number (Fig. 3a).

To explore the motif composition in GhbHLH/HLH proteins, conserved motif sites were searched by online program MEME. Fifteen conserved motifs (named motif 1-15) were identified in the GhbHLH/HLH protein sequences (Additional file 1: Figure S2). Most of the

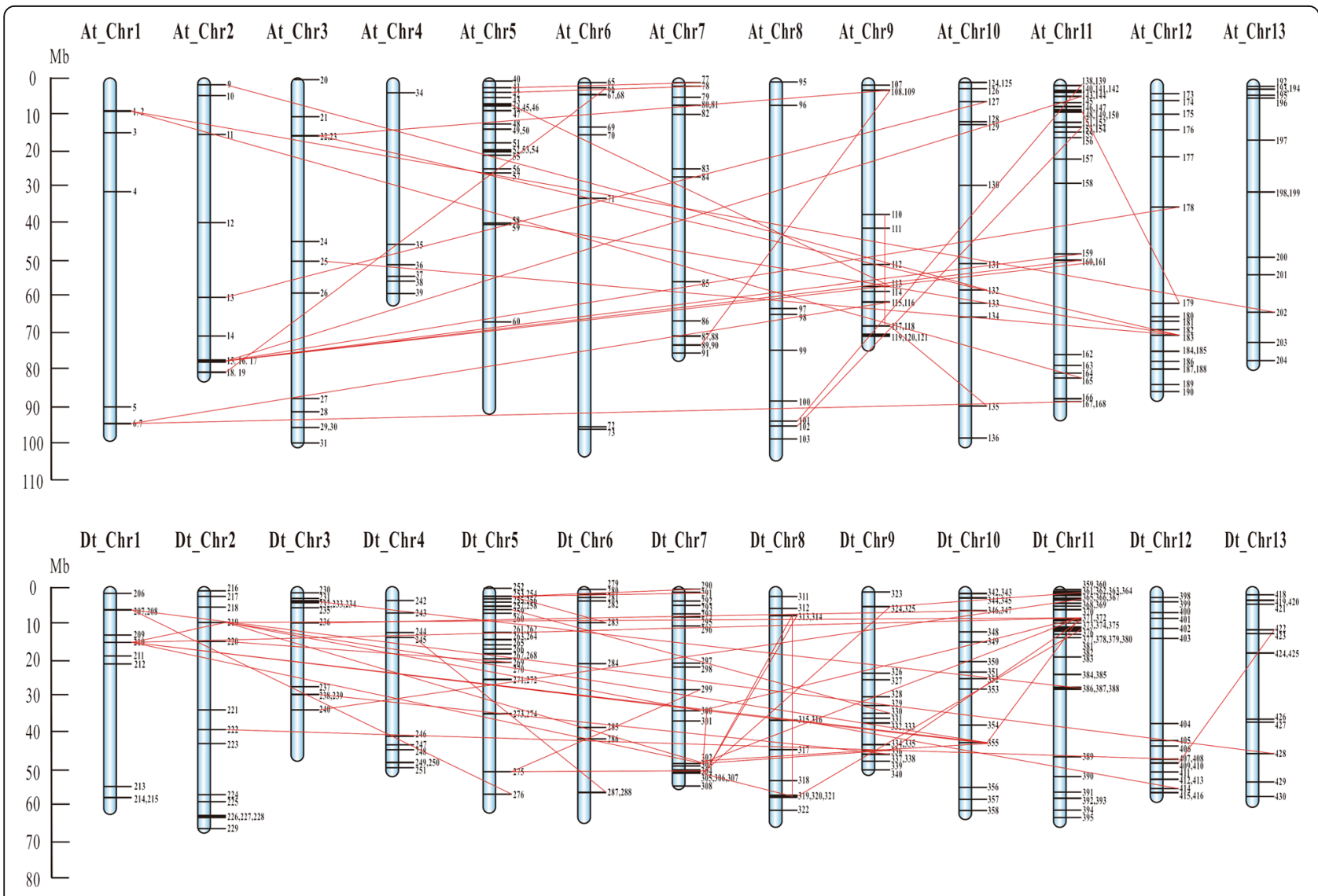

Fig. 2 Chromosomal distribution and duplication of cotton (G. hirsutum) bHLH/HLH genes. The scale is in megabases (Mb). The chromosome number is indicated at the top of each chromosome. The paralogous bHLH genes are linked with a red line 


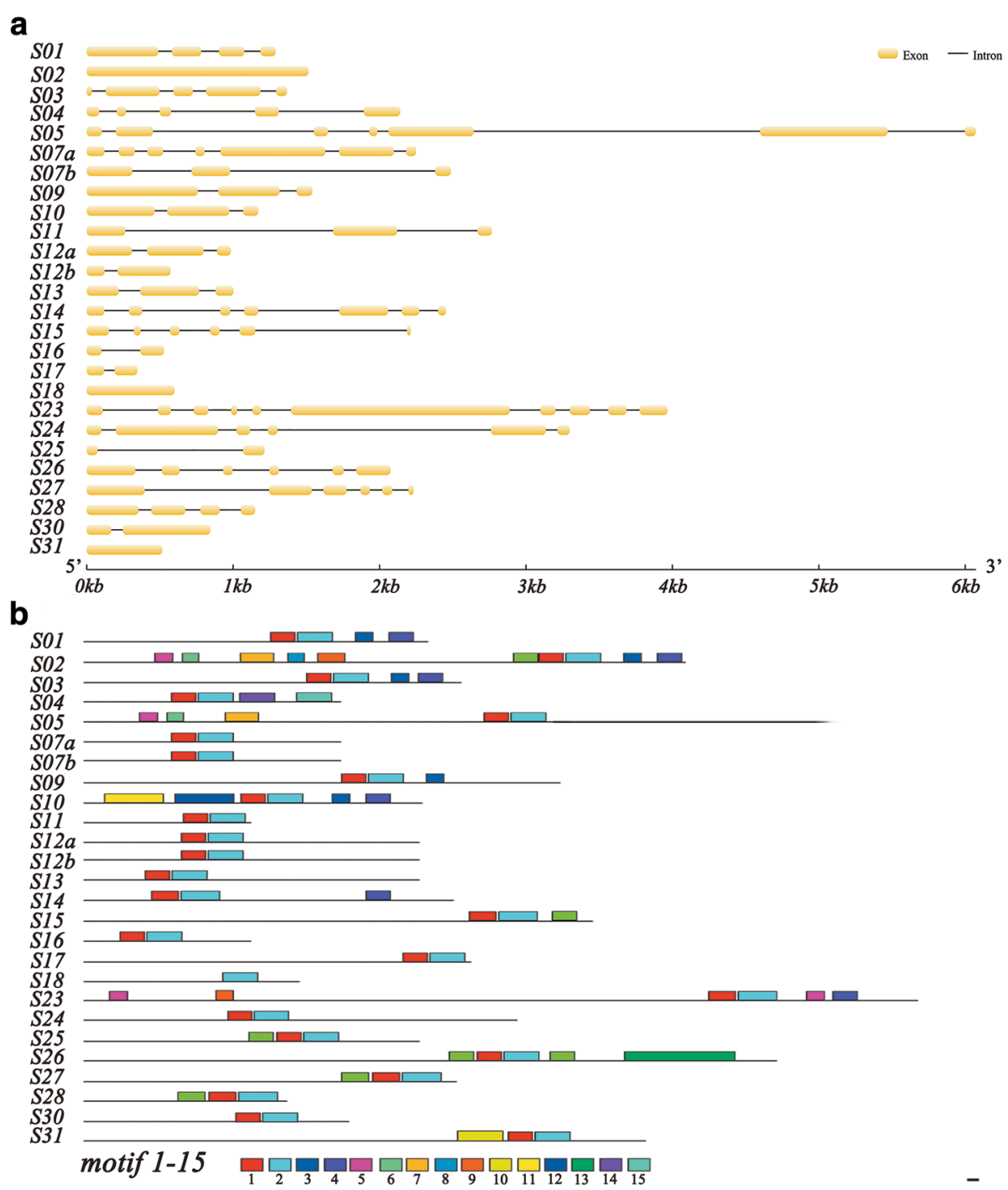

Fig. 3 Characterization of cotton (G. hirsutum) bHLH/HLH genes and their encoding proteins. a The conserved distribution of exons and introns in GhbHLH/HLH genes. The yellow columns represent exons and black lines indicate introns. b Conserved motifs of GhbHLH/HLH proteins. The motifs 1-15 were identified using MEME program, and each motif is shown with a specific color. The composition elements of all the 15 motifs are shown in Additional file 1: Figure S2

GhbHLH/HLH proteins within the same subfamily share similar motifs, while high divergence occurs among different subfamilies, indicating that the same subfamily members may perform similar roles in cotton. For instance, the bHLH/HLHs in subfamily 1 share the conserved motif $1,2,3$ and 4, while the members in subfamily 5 contain the conserved motifs $1,2,5,6$ and 7. Besides, some subfamilies have specific motifs particularly (e.g. motif 15 for subfamily 4 , motif 11 for subfamily 10, and motif 13 for subfamily 26), suggesting that these motifs may lead to the specific functions of the individual subfamily. Additionally, Prosite online program was employed to annotate the functions of the identified 15 motifs. The results showed that only motif 1 and motif 2 had hits for PROSITE motifs in the database. Both of the motifs were annotated as conserved HLH domain and were uniformly observed in all GhbHLH/HLH proteins (Fig. 3b, Additional file 1: Figure S2). Collectively, the results revealed that the exon/ intron patterns of $G h b H L H / H L H$ genes and the motif compositions of GhbHLH/HLH proteins are consistent with the phylogenetic classification of GhbHLH/HLHs in cotton.

\section{Expression of $b H L H / H L H$ genes in fibers is induced by Brassinosteroid (BR)}

To find out the candidate $b H L H / H L H$ genes involved in cotton fiber development, the public data of expression profiles of cotton genes in different organs/tissues, including ovules $(-3,0$ and $3 \mathrm{DPA})$, fibers $(5,10,20$ 
and $25 \mathrm{DPA})$, torus, stems, stamens, roots, leaves, pistils, petals and anthers were searched and analyzed for identifying the fiber preferential $b H L H / H L H$ genes. 77 $b H L H / H L H$ candidates were identified as cotton fiber preferentially expressed genes (Additional file 1: Figure S3). Subsequently, expressions of these genes were analyzed in developing fibers with 2,4-epibrassinolide (BL) and brassinazole2001 (Brz, a BR inhibitor) treatments. It has been reported that GhCPD and GhDWF4, two BR biosynthesis genes, are feedback-inhibited by BR, and expressions of these genes are significantly suppressed by exogenous BL and promoted by Brz [35, 36]. To test if the above experiments are effective, we firstly analyzed the expression levels of GhCPD and GhDWF4 in fibers with BL and Brz treatments. Our results showed that the transcripts of GhCPD and GhDWF4 were decreased observably by five folds in 3-h cultured ovules with the treatment of $100 \mathrm{nM}$ BL relative to the controls, while increased dramatically by three folds in 3-h cultured ovules with the treatment of $100 \mathrm{nM} \mathrm{Brz}$ compared with the controls (Fig. 4). Subsequently, the
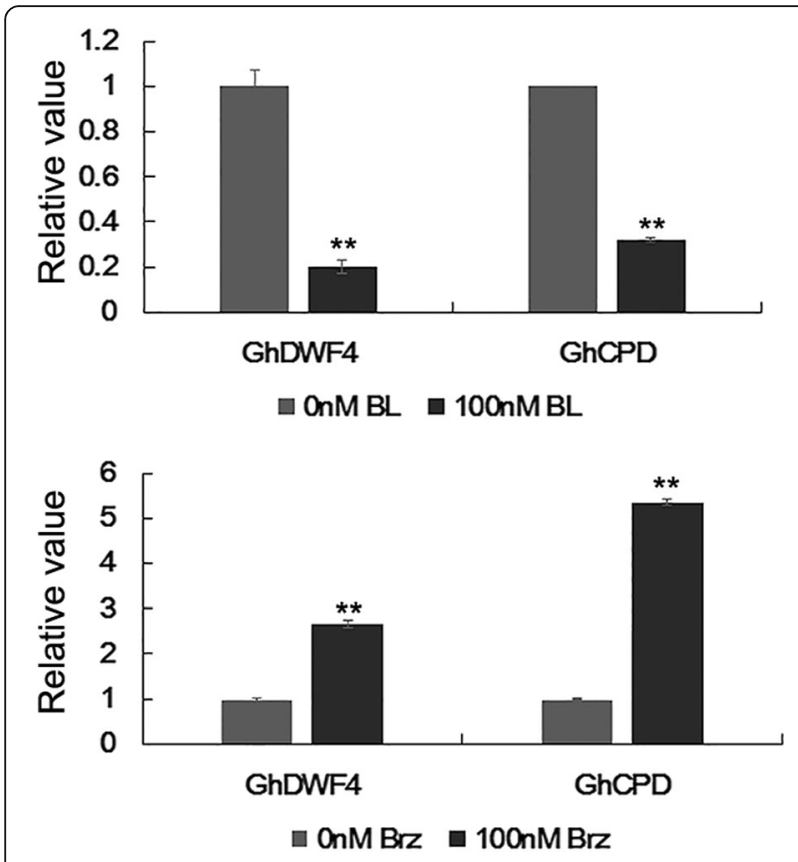

Fig. 4 Quantitative RT-PCR analysis of expression of GhCPD and GhDWF4 genes in cotton fibers treated with BL and Brz. Cotton bolls (9 DPA) were in vitro cultured in liquid BT medium without or with $100 \mathrm{nM} \mathrm{2,4-epibrassinolide} \mathrm{(eBL)} \mathrm{or} \mathrm{brassinazole2001} \mathrm{(Brz)} \mathrm{at} 30^{\circ} \mathrm{C}$ in darkness for $3 \mathrm{~h}$. Transcript levels of GhCPD and GhDWF4 were determined by quantitative RT-PCR, using GhUBl1 (EU604080) as a quantification control, and expression levels of the genes in the untreated samples ( $0 \mathrm{nM}$ BL or Brz) were set to 1. Data were processed with Microsoft Excel. Mean values and standard deviations are shown from three independent experiments. Two asterisks represent there was very significant difference $(P<0.01)$ in gene expression level between the treated sample and the untreated sample (control). DPA, day post anthesis expression levels of $77 \mathrm{bHLH} / \mathrm{HLH}$ candidates were analyzed in cotton fibers with treatments of $100 \mathrm{nM} \mathrm{BL}$ and $100 \mathrm{nM} \mathrm{Brz}$, respectively. As shown in Fig. 5, expression levels of $59 \mathrm{bHLH} / \mathrm{HLH}$ genes were altered in fibers under the treatment of $\mathrm{BL}$ or Brz. Among these candidates, $27 \mathrm{bHLH} / \mathrm{HLH}$ genes showed their moderately increased expression levels in fibers with BL treatment as well as moderately decreased expression levels in fibers with Brz treatment. Strikingly, expressions of seven genes (GhbHLH059/086/096/149/206/306/313) were largely up-regulated in fibers treated with $100 \mathrm{nM}$ $\mathrm{BL}$, and down-regulated in fibers with the treatment of $100 \mathrm{nM} \mathrm{Brz}$, suggesting that these BL-induced bHLH/ $H L H$ genes may act as regulators in cotton fiber development by responding to BR signaling. The other bHLH/HLH genes showed the decreased expression levels in fibers with BL treatment and the increased expression levels in fibers with Brz treatment. Thus, the results suggested that a number of GhbHLH/HLH genes may be involved in response to $\mathrm{BR}$ signaling for regulating fiber development of cotton.

\section{The $b H L H / H L H$ genes are differentially expressed during fiber developmental stages}

To further clarify the expression profiles of the BR-responsive $b H L H / H L H$ genes in cotton fibers, quantitative RT-PCR (qRT-PCR) was performed to detect expression levels of the identified candidate $b H L H / H L H$ genes in fibers at different developmental stages $(3,6,9,12,15,18$, and 21 DPA). As shown in Fig. 6, most of the identified $b H L H / H L H$ genes displayed relatively higher expression levels from 3 to 15 DPA fibers, while the transcripts of a few genes were accumulated in 18 to 21 DPA fibers. For example, GhbHLH021/109/150/319/428 were expressed at relatively high expression levels in 3 DPA fibers, suggesting that they may regulate very early fiber elongation, and perhaps fiber initiation, of cotton. On the other hand, the transcripts of GhbHLH004/059/086/148/195/241/282/ $336 / 352 / 373 / 400 / 421$ were accumulated mainly in $9-15$ DPA fibers, indicating they may participate in fiber development at rapid cell elongation stage. Besides, high expression levels of GhbHLHO20/102/256/351/354 genes were only found in 18-21 DPA fibers, implying that they may function in regulating secondary cell wall biosynthesis and deposition during cotton fiber development. In total, the above results suggested that GhbHLH/HLH transcription factors may play important roles in regulating cotton fiber development possibly through modulating BR signaling in fiber cells.

\section{GhbHLH282 plays a role in regulating plant growth by participating in response to $B R$ signaling}

To further investigate the roles of GhbHLH/HLH transcription factors in cotton fiber development, we chose 


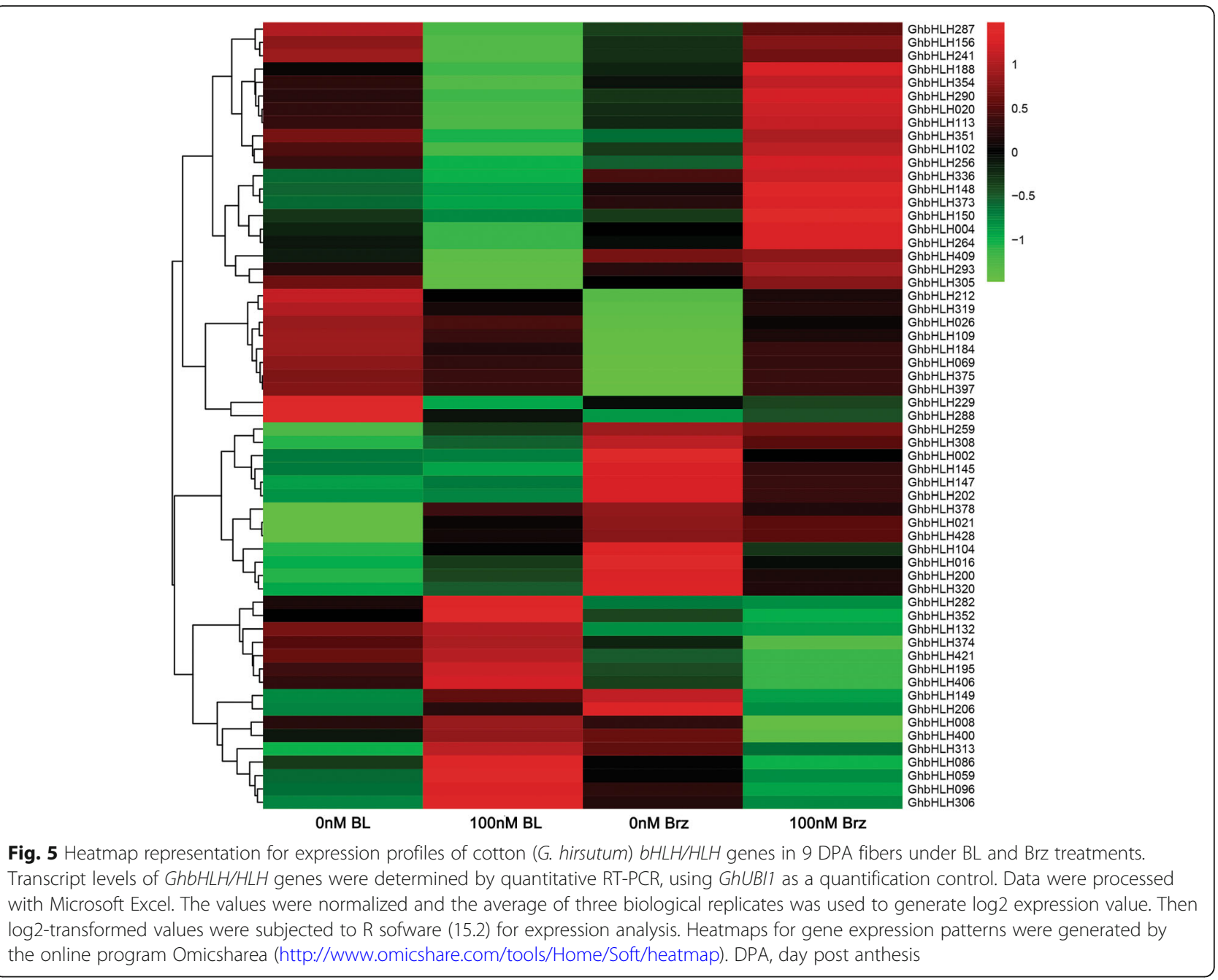

GhbHLH282 as a candidate gene that is preferentially expressed in 9-15 DPA fibers of cotton and may be related to BR signaling. Firstly, we assayed the subcellular localization of GhbHLH282 protein by employing the eGFP marker (GhbHLH282:eGFP fusion, see Methods). As shown in Fig. 7a, b and c, both the GFP fluorescence and DAPI staining were strongly accumulated in nuclei of tobacco foliar epidermis cells, suggesting that GhbHLH282 proteins were localized in the cell nucleus. Furthermore, we generated transgenic Arabidopsis plants overexpressing GhbHLH282 gene, and selected three lines (GhbHLH282OX-1, 4 and 6) with different expression levels for further study. RT-PCR analysis revealed that GhbHLH282 was expressed in the three transgenic lines and its transcripts were not detected in wild type (Fig.7d). It has been reported that BRs regulate root growth in a dose-dependent manner [17]. BRs also promoted the growth of petioles and cotyledons [24]. After 7 days incubation under light, we observed the phenotypes of the GhbHLH282 transgenic lines and wild type. The transgenic seedlings showed longer petioles and roots compared with wild type controls (Fig.7e). Statistical analysis indicated that petiole length of GhbHLH282OX transgenic seedlings was significantly increased by approximately one-fold, and root length of the transgenic lines was significantly increased by nearly $40 \%$ relative to those of wild type (Fig.7g and h). When treated with 2,4-epibrassinolide (BL), the length of petioles was increased, whereas the length of roots was reduced in both wild type and the transgenic lines. When the concentration of $\mathrm{BL}$ reached $1000 \mathrm{nM}$, there was no significant phenotypic difference between the transgenic lines and wild type (Fig.7f). These results indicated that overexpression of GhbHLH282 in Arabidopsis rendered plants insensitive to BR-induced petioles growth promotion (Fig.7g). On the contrary, overexpression of GhbHLH282 also rendered plants hyposensitive to BR-induced root growth inhibition (Fig.7h). Besides, we also observed the hypocotyls of the transgenic plants and wild type under the dark. We found that dark-grown GhbHLH282OX seedlings didn't show significant difference with wild type. Collectively, 


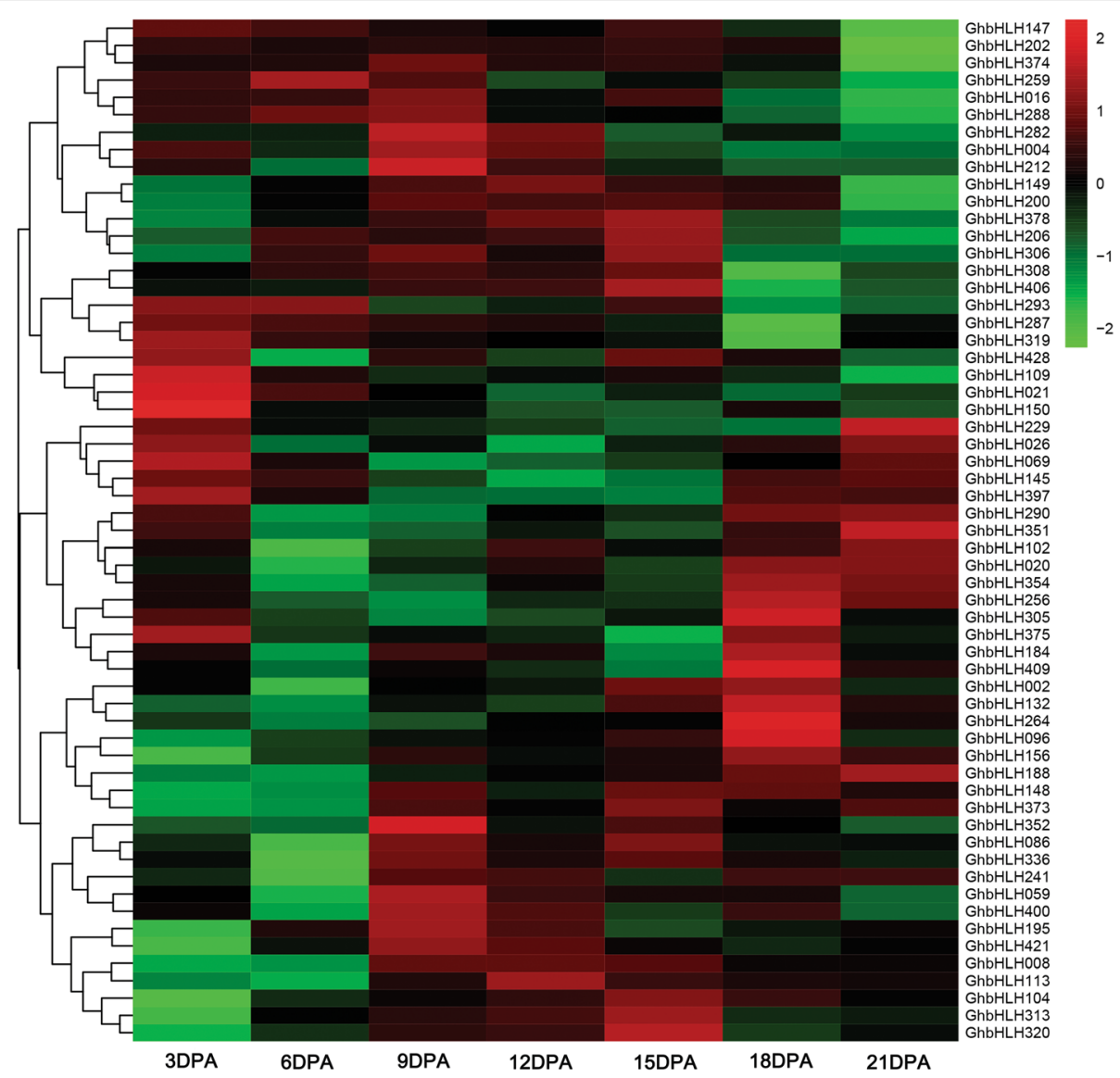

Fig. 6 Heatmap representation for expression profiles of cotton (G. hirsutum) bHLH/HLH genes in cotton fibers. Transcript levels of the $b H L H / H L H$ genes were determined by quantitative RT-PCR, using GhUB/1 as a quantification control. Data were processed with Microsoft Excel. The values were normalized and the average of three biological replicates was used to generate log2 expression value. Then log2-transformed values were subjected to R sofware (15.2) for expression analysis. Heatmaps for gene expression patterns were generated by the online program Omicsharea (http://www.omicshare.com/tools/Home/Soft/heatmap). 3DPA - 21DPA, 3 to 21 DPA (day post anthesis) fibers of cotton

the above data suggested that GhbHLH282 may play different roles in BR-promoted petioles growth and BR-inhibited roots growth.

\section{Discussion}

It is quite difficult to identify all the bHLH/HLH protein genes completely in a certain genome, especially in allotetraploid upland cotton (G. hirsutum) genome. Besides, the diversity and complexity of $b H L H / H L H$ gene families caused the imperfection of GhbHLH/HLH genes identification. In previous study, a set of cotton bHLH/HLH reference genes containing 289 paralogs were identified from annotated genomes of G. raimondii and G. arboreum [37]. In our present study, we identified $437 \mathrm{bHLH} / \mathrm{HLH}$ transcription factor genes from just-completed allotetraploid G. hirsutum genome annotation, including 205 genes in A-subgenome and 232 genes in D-subgenome. The total number of GhbHLH/HLH genes is larger than that of Arabidopsis (167) and rice (177) reported [34]. The number of
bHLH/HLH genes in upland cotton is nearly 2.6 times of that in Arabidopsis, which is consistent with the fact that the protein coding genes $(66,434$ genes) in cotton genome is about 2.6 times of that in Arabidopsis $(25,498$ genes) $[32,38]$. It is found that many bHLH/ $H L H$ genes in Arabidopsis have two or more counterparts in upland cotton, indicating that the amplification of $b H L H / H L H$ genes in upland cotton may be caused by gene duplication events. Previous studies reported that the expansion of gene families is probably due to gene duplication events, consisting of tandem duplication, segmental duplication, whole genome duplication and transposition events $[39,40]$. Our results suggested that the segmental duplication may be a major duplication event and contribute most to the amplification of $b H L H / H L H$ genes in upland cotton.

Brassinosteroid (BR) is not only involved in seed germination, vascular development and senescence of tissues, but also related to cell expansion and division [41-44]. For example, BIL4 regulates cell elongation via 


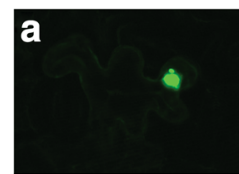

GFP

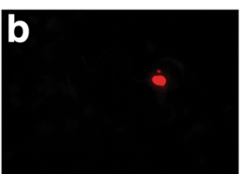

DAPI

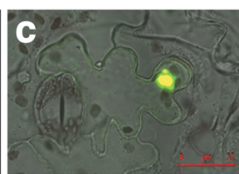

Merged

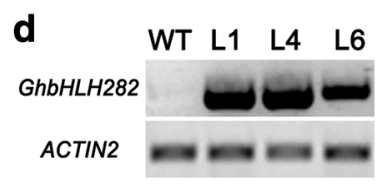

L1

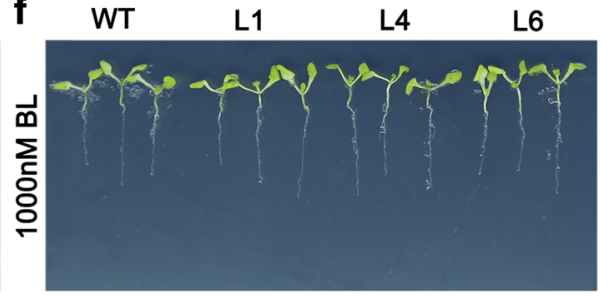

h

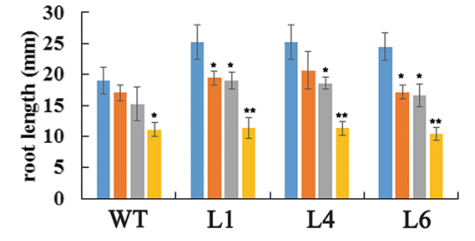

$\approx 0 \mathrm{nM} \mathrm{BL} \approx 10 \mathrm{nMBL}=100 \mathrm{nMBL} \approx 1000 \mathrm{nMBL}$

Fig. 7 Phenotypic analysis of GhbHLH282-overexpressing transgenic Arabidopsis plants in responses to 2, 4-epibrassinolide (BL). a-c Subcellular localization of GhbHLH282 protein. a Fluorescence of eGFP:GhbHLH282 fusion proteins in the tobacco (Nicotiana Benthamiana) foliar epidermis cells. $\mathbf{b}$ Nuclear DAPI staining of the same cell in a. $\mathbf{c}$ The images of a and b were merged over the bright-field. $\mathbf{d}$ Semiquantitative RT-PCR analysis of GhbHLH282 expression in the transgenic plants and wild type controls. Transcript levels of GhbHLH282 were determined by Semiquantitative RT-PCR analysis, using AtACTIN2 as a quantification control. e The phenotypes of GhbHLH282-overexpressing seedlings and wild type grown on half-strength MS medium without BL for 7 days. $\mathbf{f}$ The phenotypes of GhbHLH282-overexpressing seedlings and wild type grown on half-strength MS medium with $1000 \mathrm{nM}$ BL for 7 days. g Statistical analysis of petiole length of the GhbHLH282-overexpressing transgenic lines and wild type. The petiole growth of GhbHLH282-overexpressing plants showed the decreased sensitivity to BL, compared with mock control 0 $\mathrm{nM} \mathrm{BL}$ ), when treated with 10, 100 and $1000 \mathrm{nM} \mathrm{BL}$. h Statistical analysis of root length of the GhbHLH282-overexpressing transgenic lines and wild type. The root growth of GhbHLH282-overexpressing plants showed the increased sensitivity to BL, compared with mock control (0 nM BL), when treated with 10, 100 and $1000 \mathrm{nM}$ BL. Mean values and standard deviations are shown from three independent experiments. One or two asterisks represent there was significant $(P<0.05)$ or very significant $(P<0.01)$ difference in petiole length or root length between the BL-treated sample and the untreated sample (control), respectively. WT, wild type; L1, L4 and L6, three GhbHLH282 transgenic lines

modulating BRI1 localization for BR signal transduction [45]. Exogenous BR application inhibits root elongation and promotes the lateral root initiation [46, 47]. Besides, in vitro studies suggested that pollen tube elongation could depend partly on BR [48]. Unlimitedly, BR is also required for normal cotton fiber cell development $[26,27]$. A recent study indicated that BR signaling promotes fiber maturation of cotton through cellulose deposition in secondary cell walls [49]. PAG1, a cotton brassinosteroid catabolism gene, modulates fiber elongation via controlling the level of endogenous bioactive BR [50]. Also, our previous study revealed that Gh14-3-3 proteins regulate fiber initiation and elongation by modulating BR signaling [28]. These data indicate BR signaling plays an essential role in fiber development of cotton.

It has been reported that bHLH/HLH proteins as important regulators participate in BR signaling pathway. For instance, AtBIM2 participates in BR signaling by mediating the BR-regulated genes expression [51]. AtAIF4 is a negative regulator of BR signaling.
Overexpression of AtAIF4 results in dwarf transgenic plants, resembling BR mutants [16]. AtPREs and AtCIB3/5 are positive regulators in promoting cell elongation by modulating $\mathrm{BR}$ signaling [23, 24]. Besides, AtbHLH001 (GL3) and AtbHLH002 (EGL3) positively regulate the initiation of trichomes in Arabidopsis [11, 52]. In this study, expression of GhbHLH200 was induced by BL treatment, and its transcripts were accumulated in 6-18 DPA fibers, indicating it is probably involved in cotton fiber elongation. Expression of GhbHLH109 and GhbHLH319 was inhibited by BL and induced by Brz, and its transcripts were predominantly accumulated in very early developing ovules and fibers, suggesting that these genes may have important roles in fiber initiation. Furthermore, the transcripts of GhbHLH282 were accumulated mainly in 9-15 DPA fibers, and its expression was inhibited by BL but induced by Brz. Overexpressing GhbHLH282 not only promoted plant growth, but also changed plant sensitivity to $\mathrm{BL}$ signaling, suggesting that GhbHLH282 may play an important role in regulating 
cotton fiber development via BR signaling pathway. Collectively, we identified 59 BR responsive GhbHLH/ $H L H$ genes, which are differentially expressed during fiber initiation, elongation and secondary cell wall biosynthesis stages, implying these GhbHLH/HLH genes may participate in response to BR signaling for regulating cotton fiber development.

\section{Conclusions}

In summary, we performed a systematic analysis of the bHLH/HLH gene family in upland cotton (G. hirsutum) genome, including gene classification, phylogenetic relationship, chromosomal distribution, gene expansion, gene structure, and motif composition, as well as gene expression pattern in fiber development and in response to BR signaling. Furthermore, our results revealed the role of GhbHLH282 in plant growth possibly via BR signaling pathway. Thus, the data reported here may facilitate a more comprehensive understanding of the specific roles of the $b H L H / H L H$ genes in fiber development of cotton.

\section{Methods}

\section{Plant materials}

Upland cotton (Gossypium hirsutum) cultivar Coker312 was used in this study. Cotton plants grew in the trial field located at campus of Cental China Normal University, Wuhan, China. Flowers were tagged on the day of anthesis. Cotton bolls were harvested from the plants at $0,3,6,9,12,15,18$ and 21 days post anthesis (DPA), respectively. Ovules and fibers at different stages were removed from the collected bolls carefully. All collected materials were frozen immediately in liquid nitrogen and stored at $-80^{\circ} \mathrm{C}$ until RNA extraction.

Arabidopsis thaliana Columbia (Col-0) ecotype was used as the wild type for generating the transgenic plants. Seven-day-old seedlings grown on a half-strength MS agar medium were used as the experimental materials.

Both cotton and Arabidopsis seeds used in this study were provided by our lab.

\section{Identification of GhbHLH/HLH transcription factors}

To identify $b H L H / H L H$ genes in upland cotton, all published $b H L H / H L H$ gene sequences of Arabidopsis and rice were employed as queries to perform homologous blast searches against the upland cotton (G. hirsutum) genome databases (https://www.cottongen.org/tools/ blast/blast) [32]. Furthermore, the conserved domains of predicted bHLH/HLH proteins were evaluated using the hmmscan program and the pfam tools (http:// www.ebi.ac.uk/Tools/hmmer/search/hmmscan) according to reported methods [33].

\section{Phylogenetic analysis}

Multiple sequence alignments were conducted on the conserved bHLH/HLH domains of the identified $\mathrm{bHLH} / \mathrm{HLH}$ protien sequences in upland cotton, Arabidopsis and rice genomes by Muscle aligin method using MEGA 6.0 software. Based on the results of multiple sequence alignment, MEGA 6.0 software was employed to construct an unrooted phylogenetic tree based on alignments using the NeighborJoining (NJ) method with the following parameters: No. of differences model, pairwise deletion and 1000 bootstraps [53]. Additionally, a separate phylogenetic tree was constructed with all the conserved domains of GhbHLH/HLH protein sequences for further analysis. Then the unrooted phylogenetic tree was subjected to ITOL (http://itol.embl.de/upload.cgi) to form the interactive tree.

\section{Analysis of chromosomal distribution and duplication of genes}

To get the information about the physical locations of all $G h b H L H / H L H$ genes on chromosomes, blastn searches against upland cotton (G. hirsutum) genome databases (https://www.cottongen.org/tools/blast/blast) were employed. Then all the $G h b H L H / H L H$ genes were mapped on the chromosomes using CorelDRAW $\times 7$ software except for the scaffolds genes. The GhbHLH/ $H L H$ gene duplication events were detected according to the criteria described in previous studies [54, 55]. Paralogous $b H L H / H L H$ gene pairs were obtained based on alignment results.

\section{Assay of gene structure and conserved motifs}

The GhbHLH/HLH gene sequences and CDS sequences were identified from upland cotton (G. hirsutum) genome databases described above and were loaded into gene structure display server program (http://gsds.cbi.pku.edu.cn/) to infer the exon/intron organization of bHLH/ $H L H$ genes. To identify the conserved protein motifs, the bHLH/HLH protein sequences were submitted to online Multiple Expectation maximization for Motif Elicitation (http://meme-suite.org/tools/meme) program. The optimized MEME parameters are as follows: any number of repetitions, the optimum width from 6 to 100. The identified protein motifs were further annotated with ScanProsite.

Analysis of gene expression based on transcriptome data To uncover the GhbHLH/HLH gene expression patterns in cotton different tissues, the RPKM (reads per kb per million reads) values which denoted the expression levels of bHLH/HLH genes were obtained from a comprehensive profile of the TM-1 transcriptome data (Accession codes, SRA: PRJNA248163, http://www.ncbi.nlm.nih.gov/sra/?ter $\mathrm{m}=$ PRJNA248163). 


\section{In vitro culture of cotton ovules}

Bolls at 9 DPA (day post anthesis) from cotton plants were surface sterilized with $70 \%$ ethanol for $1 \mathrm{~min}$, followed by washing with sterile water. Ovules were picked out from these sterilized bolls, and cultured in BT liquid medium containing $5 \mu \mathrm{M}$ NAA and $0.5 \mu \mathrm{M}$ GA3 [56], supplemented with $0 \mathrm{nM}$ (control), $10 \mathrm{nM}$, $100 \mathrm{nM}$ and $1000 \mathrm{nM}$ 2,4-epibrassinolide (BL) or brassinazole2001 (Brz) at $30{ }^{\circ} \mathrm{C}$ in dark for $3 \mathrm{~h}$. Then Ovules and fibers were removed from the collected bolls carefully. All collected materials were frozen immediately in liquid nitrogen and stored at $-80^{\circ} \mathrm{C}$ until RNA extraction. Experiments were repeated at least three times and ran in three replicates each time.

\section{RNA isolation and RT-PCR analysis}

Total RNA was extracted from collected materials using Tiangen RNAprep Pure Plant Kit according to the manufacturer's instructions. The total of $2 \mu \mathrm{g}$ RNA was employed as the template for the synthesis of cDNA first-strands using M-MLV reverse transcriptase (Promega, Madison, WI) according to the manufacturer's instructions. Gene expression levels were analyzed by real-time PCR using the fluorescent intercalating dye SYBR-Green in a detection system. The expression values of $b H L H / H L H$ genes tested were normalized with an internal reference polyubiquitin gene (GhUBI1, access number in GenBank: EU604080). The relative expression levels $(R)$ were calculated using the following equation: $R=2^{-(\mathrm{Ct} 1-\mathrm{Ct} 2)}$, where $\mathrm{Ct} 1$ refers to the Ct value of $b H L H /$ $H L H$ genes while $\mathrm{Ct} 2$ is the $\mathrm{Ct}$ value of the reference gene. RT-PCR data are mean values and standard deviations (bar) of three independent experiments with three biological replicates. Based on the values of relative expression levels, heatmaps for gene expression patterns were generated by the online program Omicshare (http://www.omicshare.com/tools/Home/Soft/heatmap).

Gene expression levels in wild type and transgenic Arabidopsis were analyzed by semiquantitative RT-PCR analysis using the Hieff ${ }^{\text {mi }}$ PCR Master Mix in 96 Well Thermal Cycler (Applied Biosystems). The expression levels of GhbHLH282 gene were tested after cDNAs were normalized with an internal reference gene Arabidopsis ACTIN2 (AF428330).

\section{Generation of transgenic Arabidopsis plants}

The coding sequence of GhbHLH282 was PCR-amplified using proofreading Pfu DNA polymerase and subsequently subcloned into the expression vector $p C A M$ BIA-2300 under the control of the Caulifower mosaic virus $(C a M V) 35 S$ promoter. The recombinant vector construct was transferred into Agrobacterium tumefaciens GV3101 and then introduced into Arabidopsis using the floral dipping method. The transformed Arabidopsis seeds were selected on MS medium containing $50 \mathrm{mg} / \mathrm{L}$ kanamycin. Homozygous lines of T3 generations were used for phenotypic analysis.

\section{Subcellular localization of GhbHLH282 protein}

The coding sequence of GHbHLH282 was cloned into the downstream region of $e G F P$ in the expression vector $p C A M B I A-2300-35 S-e G F P$ under the control of the Caulifower mosaic virus $(\mathrm{CaMV}) 35 \mathrm{~S}$ promoter. The recombinant vector $p C A M B I A-2300-35 S-e G F P-G h b H L H 282$ was transferred into Agrobacterium tumefaciens GV3101 and then introduced into fully expanded leaves of tobacco ( $\mathrm{Ni}$ cotiana Benthamiana) plants using a needleless syringe. After infiltration, plants were immediately covered with plastic bags and placed at $23{ }^{\circ} \mathrm{C}$ for $48 \mathrm{~h}$, and then incubated at $28^{\circ} \mathrm{C}$ under a photoperiod of $16 \mathrm{~h}$ light $/ 8 \mathrm{~h}$ dark. Tobacco foliar epidermis were stained with 4'6-diamidino-2-phenylindole (DAPI, a nucleus-specific dye) for 1 min at room temperature before observation of GFP fluorescence and DAPI staining under the confocal fluorescence microscope (Leica, Germany). The digital images were taken and processed by SP5 software (Leica, Germany).

\section{Measurement and statistical analysis}

Arabidopsis seedlings grown on a half-strength MS medium in the presence or absence of the indicated concentrations of 2,4-epibrassinolide (BL) under a photoperiod of $16 \mathrm{~h} \mathrm{light} / 8 \mathrm{~h}$ dark at $22^{\circ} \mathrm{C}$ were photographed, and petiole and root length of these seedlings was measured using the Image J software (https://imagej.en.softonic.com/ ). All experiments were done at a minimum in triplicate, and the data were statistically analyzed by the Student's $\mathrm{t}$-test. More than 50 seedlings were used for each biological replicate.

All primers used are listed in Additional file 1: Table S3.

\section{Additional files}

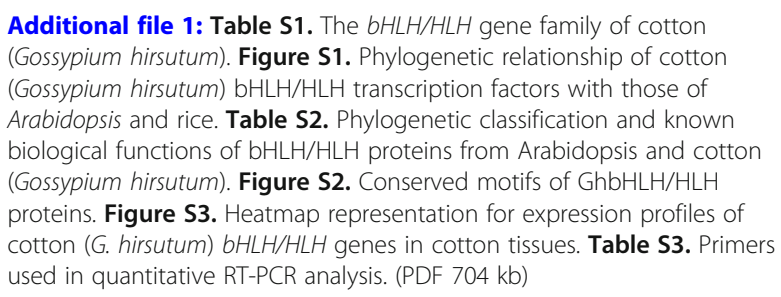

Additional file 1: Table S1. The bHLH/HLH gene family of cotton (Gossypium hirsutum). Figure S1. Phylogenetic relationship of cotton (Gossypium hirsutum) bHLH/HLH transcription factors with those of Arabidopsis and rice. Table S2. Phylogenetic classification and known biological functions of bHLH/HLH proteins from Arabidopsis and cotton (Gossypium hirsutum). Figure S2. Conserved motifs of GhbHLH/HLH proteins. Figure S3. Heatmap representation for expression profiles of cotton (G. hirsutum) bHLH/HLH genes in cotton tissues. Table S3. Primers used in quantitative RT-PCR analysis. (PDF $704 \mathrm{~kb}$ )

\section{Acknowledgements}

Not applicable.

\section{Funding}

This work was supported by the National Natural Sciences Foundation of China (Grant No. 31671255), and the National Key R\&D Program of China (Grant No. 2016YFD0100505). 


\section{Availability of data and materials}

All data used during the current study are included in this published article or are available from the corresponding author on reasonable request.

\section{Authors' contributions}

$X B L$ and $R L$ conceived and designed the research, $R L, J Z, D L, Y L W$ and $Y W$ performed the experiments, RL and XBL analyzed data and wrote the paper. All authors read and approved the final manuscript.

\section{Ethics approval and consent to participate}

Cotton is a very common crop in the world. This study does not contain any research requiring ethical consent or approval.

\section{Consent for publication}

Not applicable.

\section{Competing interests}

The authors declare that they have no competing interests.

\section{Publisher's Note}

Springer Nature remains neutral with regard to jurisdictional claims in published maps and institutional affiliations.

\section{Received: 14 August 2018 Accepted: 14 November 2018}

\section{Published online: 27 November 2018}

\section{References}

1. Wendel JF, Cronn RC. Polyploidy and the evolutionary history of cotton. Adv Agron. 2003;78:139-86.

2. Haigler $\mathrm{CH}$, Betancur L, Stiff MR, Tuttle JR. Cotton fiber: a powerful single-cell model for cell wall and cellulose research. Front Plant Sci. 2012;3(104):00104

3. Kim HJ, Triplett BA. Cotton fiber growth in planta and in vitro, models for plant cell elongation and cell wall biogenesis. Plant Physiol. 2001; 127(4):1361-6

4. Kim HJ, Hinchliffe DJ, Triplett BA, Chen ZJ, Stelly DM, Yeater KM, et al. Phytohormonal networks promote differentiation of fiber initials on preanthesis cotton ovules grown in vitro and in planta. PLoS One. 2015; 10(4):e0125046

5. Murre C, McCaw PS, Baltimore D. A new DNA binding and dimerization motif in immunoglobulin enhancer binding, daughterless, MyoD, and myc proteins. Cell. 1989;56(5):777-83.

6. Ferré-D'Amaré AR, Pognonec P, Roeder RG, Burley SK. Structure and function of the b/HLH/Z domain of USF. EMBO J. 1994;13(1):180-9.

7. Nair SK, Burley SK. Functional genomics: recognizing DNA in the library. Nature. 2000;404(4):715-8.

8. Leivar P, Quail PH. PIFs: pivotal components in a cellular signaling hub. Trends Plant Sci. 2011;16(1):19-28.

9. Heisler MG, Atkinson A, Bylstra YH, Walsh R, Smyth DRSPATULA. A gene that controls development of carpel margin tissues in Arabidopsis, encodes a bHLH protein. Development. 2001;128(7):1089-98.

10. Josse EM, Gan YB, Bou-Torrent J, Stewart KL, Gilday AD, Jeffree CE. A DELLA in disguise: SPATULA restrains the growth of the developing Arabidopsis seedling. Plant Cell. 2011;23(4):1337-51.

11. Serna L, Martin C. Trichomes: different regulatory networks lead to convergent structures. Trends Plant Sci. 2006;11(6):274-80.

12. Dai XM, Zhou LM, Zhang W, Cai L, Guo HY, Tian HN, et al. A single amino acid substitution in the R3 domain of GLABRA1 leads to inhibition of trichome formation in Arabidopsis without affecting its interaction with GLABRA3. Plant Cell Environ. 2016;39(4):897-907.

13. Zhao MZ, Morohashi K, Hatlestad G, Grotewold E, Lloyd A. The TTG1-bHLHMYB complex controls trichome cell fate and patterning through direct targeting of regulatory loci. Development. 2008;135(11):1991-9.

14. Zhao HT, Wang XX, Zhu DD, Cui SJ, L X, Cao Y, et al. A single amino acid substitution in IIIf subfamily of basic helix-loop-helix transcription factor AtMYC1 leads to trichome and root hair patterning defects by abolishing its interaction with partner proteins in Arabidopsis. J Biol Chem. 2012;287(17):14109-41.

15. Friedrichsen DM, Nemhauser J, Muramitsu T, Maloof JN, Alonso J, Ecker JR et al. Three redundant brassinosteroid early response genes encode putative bHLH transcription factors required for normal growth. Genetics. 2002:162(3):1445.

16. Wang H, Zhu Y, Fujioka S, Asami T, Li J, Li J. Regulation of Arabidopsis brassinosteroid signaling by atypical basic helix-loop-helix proteins. Plant Cell. 2009;21(12):3781-91.

17. Zhang LY, Bai MY, Wu J, Zhu JY, Wang H, Zhang Z. Antagonistic HLH/ bHLH transcription factors mediate brassinosteroid regulation of cell elongation and plant development in Rice and Arabidopsis. Plant Cell. 2009;21(12):3767-80.

18. Malinovsky FG, Batoux M, Schwessinger B, Youn JH, Stransfeld L, Win J. Antagonistic regulation of growth and immunity by the Arabidopsis basic helix-loop-helix transcription factor homolog of brassinosteroid enhanced expression2 interacting with increased leaf inclination 1 binding $\mathrm{bHLH} 1$. Plant Physiol. 2014;164(3):1443-55.

19. Kim Y, Song JH, Park SU, Jeong YS, Kim SH. Brassinosteroid-induced transcriptional repression and dephosphorylation-dependent protein degradation negatively regulate BIN2-interacting AIF2 (a BR signalingnegative regulator) bHLH transcription factor. Plant Cell Physiol. 2017; 58(2):227-39.

20. Toledo-Ortiz G, Huq E, Quail PH. The Arabidopsis basic/helix-loop-helix transcription factor family. Plant Cell. 2003;15(8):1749-70.

21. Gruszka D. The brassinosteroid signaling pathway — new key players and interconnections with other signaling networks crucial for plant development and stress tolerance. Int J Mol Sci. 2013;14(5):8740.

22. Bai MY, Fan M, Oh E, Wang ZY. A triple helix-loop-helix/basic helixloop-helix cascade controls cell elongation downstream of multiple hormonal and environmental signaling pathways in Arabidopsis. Plant Cell. 2012;24(12):4917-29.

23. Fan M, Bai MY, Kim JG, Wang T, Oh E, Chen L. The bHLH transcription factor HBI1 mediates the trade-off between growth and pathogenassociated molecular pattern-triggered immunity in Arabidopsis. Plant Cell. 2014:26(2):828-41.

24. Ikeda M, Fujiwara S, Mitsuda N, Ohme-Takagi M. A triantagonistic basic helix-loop-helix system regulates cell elongation in Arabidopsis. Plant Cell. 2012;24(11):4483-97.

25. Jang $S, A \cap G, L i H Y$. Rice leaf angle and grain size are affected by the OsBUL1 transcriptional activator complex. Plant Physiol. 2016;173(1):688-702

26. Sun $Y$, Veerabomma S, Abdel-Mageed HA, Fokar M, Asami T, Yoshida S, et al. Brassinosteroid regulates fiber development on cultured cotton ovules. Plant Cell Physiol. 2005;46(8):1384-91.

27. Shi YH, Zhu SW, Mao XZ, Feng JX, Qin YM, Zhang L, et al. Transcriptome profiling, molecular biological, and physiological studies reveal a major role for ethylene in cotton fiber cell elongation. Plant Cell. 2006;18(3):651-64.

28. Zhou Y, Zhang ZT, Li M, Wei XZ, Li XJ, Li BY, et al. Cotton (Gossypium hirsutum) 14-3-3 proteins participate in regulation of fibre initiation and elongation by modulating brassinosteroid signalling. Plant Biotechnol J. 2015:13(2):269-80.

29. Samuel YS, Cheung F, Lee JJ, Ha M, Wei NE, Sze SH, et al. Accumulation of genome-specific transcripts, transcription factors and phytohormonal regulators during early stages of fiber cell development in allotetraploid cotton. Plant J. 2006:47(5):761-75.

30. Yuan DJ, Tu LL, Zhang XL. Generation, annotation and analysis of first largescale expressed sequence tags from developing fiber of Gossypium barbadense L. PLoS One. 2011;6(7):e22758.

31. Liu K, Sun J, Yao LY, Yuan YL. Transcriptome analysis reveals critical genes and key pathways for early cotton fiber elongation in Ligon lintless-1 mutant. Genomics. 2012;100(1):42-50

32. Zhang TZ, Hu Y, Jiang W, Fang L, Guan X, Chen J, et al. Sequencing of allotetraploid cotton (Gossypium hirsutum L. acc. TM-1) provides a resource for fiber improvement. Nat Biotechnol. 2015:33(5):531-7.

33. Finn RD, Clements J, Arndt W, Miller BL, Wheeler TJ, Schreiber F, et al. HMMER web server: 2015 update. Nucleic Acids Res. 2015:43(W1):W30-8.

34. Carretero-Paulet L, Galstyan A, Roig-Villanova I, Martínez-García JF, BilbaoCastro JR, Robertson DL, et al. Genome-wide classification and evolutionary analysis of the bHLH family of transcription factors in Arabidopsis, poplar, rice, moss, and algae. Plant Physiol. 2010;153(3):1398-412.

35. De GL, Vandenbussche F, Tietz O, Palme K, Van DSD. Auxin, ethylene and brassinosteroids: tripartite control of growth in the Arabidopsis hypocotyl. Plant Cell Physiol. 2005;46(6):827-36.

36. Si J, Sun $Y$, Wang LU, Qin $Y$, Wang C, Wang $X$, et al. Functional analyses of Populus euphratica brassinosteroid biosynthesis enzyme genes DWF4 
(PeDWF4) and CPD (PeCPD) in the regulation of growth and development of Arabidopsis thaliana. J Biosci. 2016;41(4):727-42.

37. Yan Q, Liu HS, Yao D, Li X, Chen H, Dou Y, et al. The basic/helix-loop-helix protein family in Gossypium: reference genes and their evolution during tetraploidization. PLoS One. 2015;10(5):e0126558.

38. Initiative AG. Analysis of the genome sequence of the flowering plant Arabidopsis thaliana. Nature. 2000;408(6814):796-815.

39. Zhang JZ. Evolution by gene duplication: an update. Trends Ecol Evol. 2003;18(6):292-8.

40. Flagel LE, Wendel JF. Gene duplication and evolutionary novelty in plants. New Phytol. 2009;183(3):557-64.

41. Steber $C M$, Mccourt $P$. A role for brassinosteroids in germination in Arabidopsis. Plant Physiol. 2001;125(2):763-9.

42. Jeong YJ, Shang Y, Kim BH, Kim SY, Song JH, Lee JS. BAK7 displays unequal genetic redundancy with BAK1 in brassinosteroid signaling and early senescence in Arabidopsis. Mol Cells. 2010;29(3):259-66.

43. Zhiponova MK, Vanhoutte I, Boudolf V, Betti C, Dhondt S, Coppens F, et al. Brassinosteroid production and signaling differentially control cell division and expansion in the leaf. New Phytol. 2013;197(2):490-502.

44. Salazarhenao JE, Lehner R, Betegónputze I, Vilarrasablasi J, Cañodelgado Al. BES1 regulates the localization of the brassinosteroid receptor BRL3 within the provascular tissue of the Arabidopsis primary root. J Exp Bot. 2016;67(17):4951-61.

45. Yamagami A, Saito C, Nakazawa M, Fujioka S, Uemura T, Matsui M, et al. Evolutionarily conserved BIL4 suppresses the degradation of brassinosteroid receptor BRI1 and regulates cell elongation. Sci Rep. 2017:7(1):5739.

46. Müssig C, Shin GH, Altmann T. Brassinosteroids promote root growth in Arabidopsis. Plant Physiol. 2003;133(3):1261-71.

47. Deng Q, Wang X, Zhang D, Wang X, Feng C, Xu S, et al. BRS1 function in facilitating lateral root emergence in Arabidopsis. Int J Mol Sci. 2017; 18(7):1549.

48. Vogler F, Schmalzl C, Englhart M, Bircheneder M, Sprunck S. Brassinosteroids promote Arabidopsis pollen germination and growth. Plant Reprod. 2014;27(3):153-67.

49. Sun Y, Veerabomma S, Fokarb M, Abidic N, Hequetc E, Payton P, et al. Brassinosteroid signaling affects secondary cell wall deposition in cotton fibers. Ind Crop Prod. 2015;65:334-42.

50. Yang ZR, Zhang C, Yang X, Liu K, Wu Z, Zhang X, et al. PAG1, a cotton brassinosteroid catabolism gene, modulates fiber elongation. New Phytol. 2014;203(2):437-48.

51. Yin Y, Vafeados D, Tao Y, Yoshida S, Asami T, Chory J, et al. A new class of transcription factors mediates brassinosteroid-regulated gene expression in Arabidopsis. Cell. 2005;120(2):249-59.

52. Morohashi K, Zhao M, Yang M, Read B, Lloyd A, Lamb R, et al. Participation of the Arabidopsis bHLH factor GL3 in trichome initiation regulatory events. Plant Physiol. 2007;145(3):736-46.

53. Tamura K, Stecher G, Peterson D, Filipski A, Kumar S. MEGA6: molecular evolutionary genetics analysis version 6.0. Mol Biol Evol. 2013;30(12):2725-9.

54. Gu Z, Cavalcanti A, Chen FC, Bouman P, Li WH. Extent of gene duplication in the genomes of Drosophila, nematode, and yeast. Mol Biol Evol. 2002;19(3):256-62.

55. Yang S, Zhang X, Yue JX, Tian D, Chen JQ. Recent duplications dominate NBS-encoding gene expansion in two woody species. Mol Gen Genomics. 2008;280(3):187-98.

56. Beasley CA, Ting IP. Effects of plant growth substances on in vitro fiber development from unfertilized cotton ovules. Am J Bot. 1974;61 (2):188-94.

Ready to submit your research? Choose BMC and benefit from:

- fast, convenient online submission

- thorough peer review by experienced researchers in your field

- rapid publication on acceptance

- support for research data, including large and complex data types

- gold Open Access which fosters wider collaboration and increased citations

- maximum visibility for your research: over $100 \mathrm{M}$ website views per year

At BMC, research is always in progress.

Learn more biomedcentral.com/submissions 